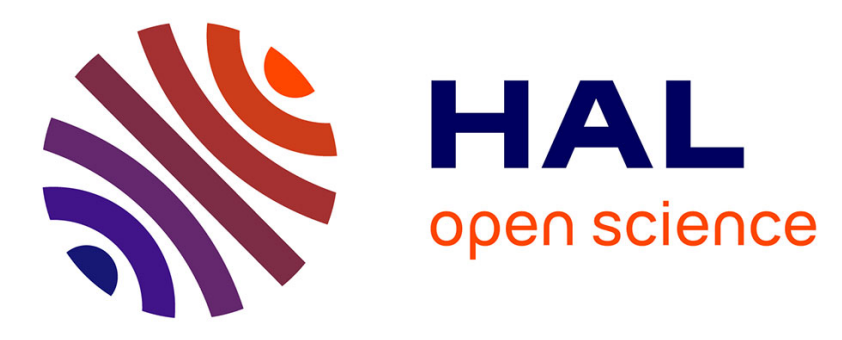

\title{
A New Histogram-based Descriptor for Images Retrieval from databases
}

\author{
Kidiyo Kpalma, Cong Bai, Miloud Chikr El-Mezouar, Kamel Belloulata, \\ Nasreddine Taleb, Lakhdar Belhallouche, Djamal Boukerroui
}

\section{- To cite this version:}

Kidiyo Kpalma, Cong Bai, Miloud Chikr El-Mezouar, Kamel Belloulata, Nasreddine Taleb, et al.. A New Histogram-based Descriptor for Images Retrieval from databases. International Workshop on Next Generation Intelligent Medical Decision Support Systems (MedDecSup 2012), Jul 2012, Sofia, Bulgaria. hal-00728214

\section{HAL Id: hal-00728214 https://hal.science/hal-00728214}

Submitted on 5 Sep 2012

HAL is a multi-disciplinary open access archive for the deposit and dissemination of scientific research documents, whether they are published or not. The documents may come from teaching and research institutions in France or abroad, or from public or private research centers.
L'archive ouverte pluridisciplinaire HAL, est destinée au dépôt et à la diffusion de documents scientifiques de niveau recherche, publiés ou non, émanant des établissements d'enseignement et de recherche français ou étrangers, des laboratoires publics ou privés. 


\title{
A New Histogram-based Descriptor for Images Retrieval from databases
}

\author{
Kidiyo KPALMA ${ }^{1, \mathrm{a}}$, Cong BAI ${ }^{\mathrm{a}}$, Miloud CHIKR EL MEZOUAR ${ }^{\mathrm{a}, \mathrm{b}}$ \\ Kamel BELLOULATA ${ }^{\mathrm{b}}$, Nasreddine TALEB ${ }^{\mathrm{b}}$, Lakhdar BELHALLOUCHE ${ }^{\mathrm{b}}$ \\ Djamal BOUKERROUI ${ }^{\mathrm{C}}$ \\ ${ }^{a} U E B$ - INSA, IETR, UMR 6164, F-35708 Rennes, France \\ ${ }^{\mathrm{b}} \mathrm{RCAM}$ - Université de Sidi Bel Abbès, Algeria \\ ${ }^{\mathrm{c}}$ Heudiasyc UMR 7253 UTC, F-60205 Compiègne, France
}

\begin{abstract}
In this paper, we propose a new approach for designing histogrambased descriptors. For demonstration purpose, we generate a descriptor based on the histogram of differential-turning angle scale space (d-TASS) function and its derived data. We then compare the proposed histogram-based descriptor with the traditional histogram descriptors in terms of retrieval performance from image databases. Experiments on three shapes databases demonstrate the efficiency and the effectiveness of the new technique: the proposed technique of histogram-based descriptor outperforms the traditional one. These experiments showed also that the proposed histogram-based descriptor using d-TASS function and the derived features performs well compared with the state-of-theart. When applied to texture images retrieval, the proposed approach yields higher performance than the traditional histogram-based descriptors. From these results, we believe that the proposed histogram-based descriptor should perform efficiently for medical images retrieval so we will focus on this aspect in the future work.
\end{abstract}

Index Terms—pattern recognition, image description, image retrieval, texture image, differential-turning angle scale space, turning angle scale space, bull's eye performance.

\section{Introduction}

With the extraordinary growth of image databases due to the development of digital systems, automatic image retrieval within large databases becomes necessary. To make them more efficient and more easy to use, retrieval systems must be based preferably on images content.

In the present study, we use the d-TASS (differential-Turning Angle Scale Space) function and two related measurements to generate their corresponding histograms

\footnotetext{
${ }^{1}$ Corresponding author: kidiyo.kpalma@insa-rennes.fr
} 
from which the shape descriptor is built. The d-TASS function introduced in [4] and [5] is known to yield characteristic properties and to be invariant under translation, rotation and scale change.

The rest of the paper is organized as follows: after a brief description of the d-TASS construction and the definition of the related features, in section 2, the proposed descriptor is presented in section 3. Section 4 presents some experimental results and compares the proposed and the traditional histogram-based descriptor generation. We also present some comparison of the proposed approach and some existing techniques of the state of the art. Finally, section 5 gives some discussions and concludes the paper.

\section{Description of the features}

As presented in [5], the input contour is defined by a set of $\mathrm{N}$ points ordered counter clockwise in the plane. Given a starting point $\mathrm{P}_{0}$, the points are numbered from $\mathrm{P}_{0}$ to $\mathrm{P}_{\mathrm{N}-1}$. The vector $\vec{V}_{n}$ originating at $\mathrm{P}_{\mathrm{n}}$ and oriented towards $\mathrm{P}_{\mathrm{n}+1}$ makes an angle $\theta_{n}$ with the $\mathrm{x}$-axis. This angle is called the turning angle. Figure 1 , illustrates the principle. From this angle, we define the differential turning angle (d-TA) function $\varphi_{n}$ :

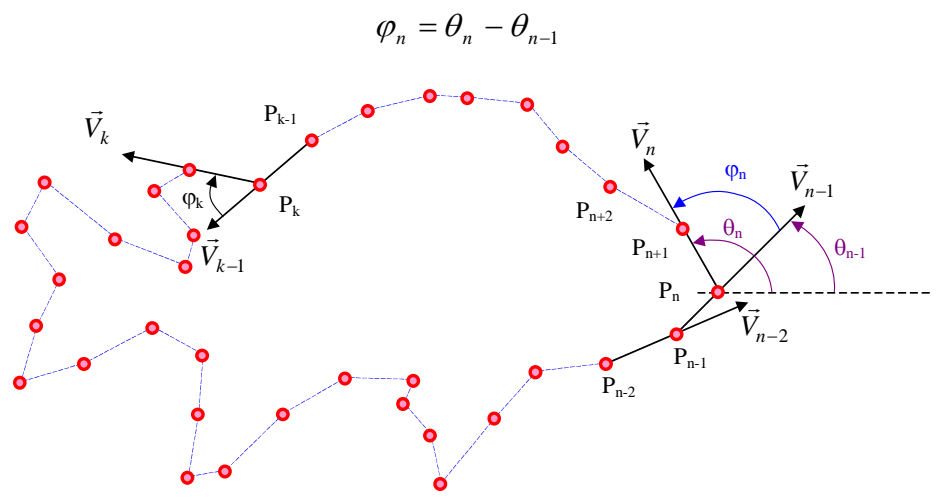

Fig.1: Illustration of the differential-turning angle

The d-TA function yields an expression of the curvature of the contour thus its zerocrossing corresponds to that obtained from curvature analysis [12] and [13].

\subsection{Generation of the d-TASS function}

By definition, the d-TASS function is generated, by progressively smoothening the contour with a Gaussian filter [4], [5] and [6]. Given a Gaussian kernel with a standard deviation $\sigma_{0}$, a progressive filtering is performed by iterating the operation so that the filtering scale $\sigma_{\mathrm{s}}=\sigma_{0} \sqrt{\mathrm{s}}$ is reached after s iterations. In this study, a Gaussian kernel of 
size 3 ( $g=[0.25,0.50,0.25])$ corresponding to $\sigma_{0}=\sqrt{2 / \pi}$ is used. After each iteration, the d-TA function is computed. Thus, given a range of scales (a range of iterations), one obtains the d-TASS function.

From the d-TA function, three essential points are derived to give the d-TASS map [5]. These essential points are:

- $\alpha$-points corresponding to local minimums of the d-TA function,

- $\beta$-points corresponding to local maximums of the d-TA function,

- $\gamma$-points corresponding to zero-crossings of the d-TA function.

\subsection{Derived features}

Generating the d-TASS function and the corresponding map is done according to the following procedure. The input contour is resampled to generate 360 equidistant points [4] and [5] ordered counter clockwise. From this input sequence, the d-TA function is computed to generate corresponding essential points. In this study, we focus on the $\gamma$ points only because they will be used to set the stop condition of the iteration process. Like in the case of curvature scale space (CSS) [12], the stop condition is that there is no more $\gamma$-point at the current scale.

At each scale $\sigma_{\mathrm{s}}$ (iteration s), $\gamma$-points are detected and the following desired features are computed:

- an area function: area of the triangle defined by the current point $\mathrm{P}_{\mathrm{n}}$, the following one $\mathrm{P}_{n+1}$ and the mid-point $\mathrm{P}_{\mathrm{M}}$ between two consecutive $\gamma$-points (see Fig.2.b).

- a distance function: distance between the current contour point $\mathrm{P}_{\mathrm{n}}$ and the midpoint $\mathrm{P}_{\mathrm{M}}$ (see Fig.2.c)

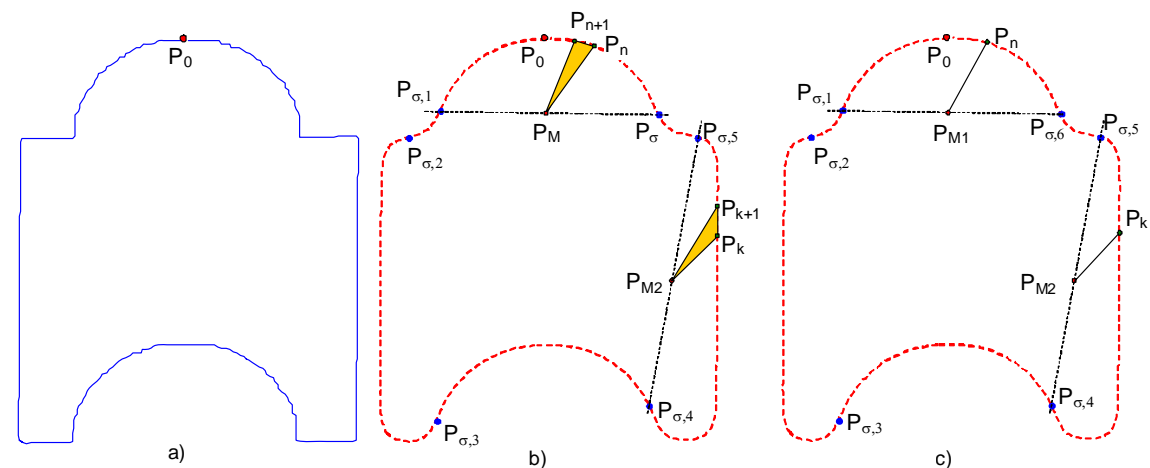

Fig.2: a: original contour and the illustration of b) the area function and c) the distance function.

These measures are characteristic of the contour segment. By iterating the process for a range of scales (until the stop condition), we obtain the area scale space function (ASSF) and the distance scale space function (DSSF), respectively, for the area 
function and the distance function. Figure 3 illustrates the three corresponding functions for the contour shown in Fig.2.a. The superimposed red curves represent the map of $\gamma$-points. On this figure, the x-axis represents the curvilinear abscissa and the $\mathrm{y}$-axis the scale (or the iteration number).
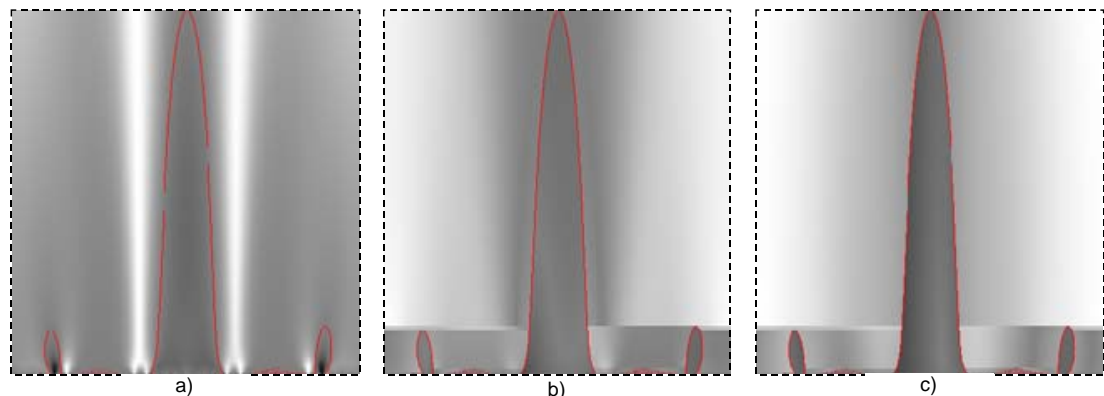

Fig.3: Illustration of a) d-TASS function, b) ASSF function and c) DSSF function.

\section{Definition of the proposed descriptor}

As said before, the proposed shape descriptor is based on the histograms of the dTASS function and the related ASSF and DSSF functions. The idea of the proposed technique of histogram generation is based on the approach used in [1] and in [7]. The histogram of the whole database is generated to produce a reference histogram from which we calculate decision levels that enable us to generate descriptors. Commonly and particularly in [7], histogram descriptors are designed by simply uniformly quantizing the feature of interest (say the image grey scale) and then counting the number of elements having the same value and then taking the number of desired bins. This approach has proved to provide efficient results.

The proposed technique is borrowed from image coding or image restoration where histogram equalization is applied. In image processing, it is shown that this adaptive quantification can help to reduce the bit rate and to enhance signal-to-noise ratio. Starting from this observation, we propose to design the histogram-based descriptor by defining the histogram bins like in the case of adaptive quantization.

First, the reference histogram of the database is generated and normalize to sum to the unity. Then the adaptive quantization is designed such a way that the "probability" to get a value between any two consecutive decision levels is equal. Knowing the desired number $\mathrm{K}$ of bins, this probability equals $\mathrm{PK}=1 / \mathrm{K}$. This enables to generate decision levels that are then used to define the descriptor for the query. Finally, for each individual histogram, use the generated decision levels and sum up the bins between two consecutive levels. This leads to the descriptor for the contour under study. Figure 4, illustrates the principle and indicates the decision levels; it also shows an example of generated histogram bins. 
The proposed descriptor is thus defined by the concatenation of individual descriptors, corresponding to the three features, as follows:

$$
\mathrm{V}=\left[\mathrm{H}_{\mathrm{dTASS}} \mathrm{H}_{\mathrm{ASSF}} \mathrm{H}_{\mathrm{DSSF}}\right]
$$

where $\mathrm{H}_{\mathrm{dTASS}}, \mathrm{H}_{\mathrm{ASSF}}$ are $\mathrm{H}_{\mathrm{DSSF}}$ are the respective generated histograms descriptors of d-TASS, ASSF and DSSF functions. Thus the length of the generated descriptor V is $\mathrm{D}=3 \mathrm{~K}$

a)

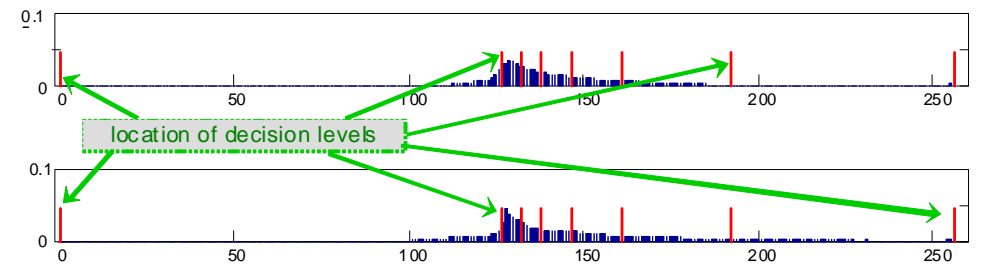

c) |l!!!!!

Fig.4: a) Reference histogram of the whole database, b) histogram of a contour ("bonefishes"), and c) its 7-bins' proposed histogram.

\section{Evaluation of the proposed descriptor}

To evaluate the ability of the proposed descriptor to discriminate objects, we have conducted experiments of image retrieval from database of planar objects. In order to compare our results with those from other methods, the experiments are done on three well-known databases that are the kimia99 dataset [8], the multiview curve dataset (MCD) [10], [11] and that extracted from the SQUID marine animals [12]. The performance of the descriptors is evaluated using the Precision-Recall curve averaged over the whole database and particularly we present the bull's eye performance (BEP). The similarity measure is based on Manhattan distance. As proposed in [12], some global parameters like eccentricity, aspect ratio and circularity are taken into account. Based on this, a two-steps hierarchical procedure is performed: given a global parameter $\mathrm{g}$, a threshold th $\mathrm{h}_{\mathrm{g}}$ is set so that all models giving $\frac{\left|\mathrm{g}_{\mathrm{g}}-\mathrm{g}_{\mathrm{M}}\right|}{\max \left(\mathrm{g}_{\mathrm{g}}, \mathrm{g}_{\mathrm{g}}\right)} \leq \mathrm{th}_{\mathrm{g}}<1$ are discarded from the retrieval procedure, where $\mathrm{g}_{\mathrm{Q}}$ and $\mathrm{g}_{\mathrm{M}}$ are the parameter corresponding, respectively, to query Q and model M.

Then we define the similarity measure between the query and a model, represented by their respective descriptor vectors $\mathrm{V}_{\mathrm{Q}}$ and $\mathrm{V}_{\mathrm{M}}$, as follows:

$$
\operatorname{Sim}(\mathrm{Q}, \mathrm{M})=\alpha \sum_{\mathrm{i}=1}^{\mathrm{G}}\left|\mathrm{g}_{\mathrm{Q}}(\mathrm{i})-\mathrm{g}_{\mathrm{M}}(\mathrm{i})\right|+\beta \sum_{\mathrm{i}=1}^{\mathrm{D}}\left|\mathrm{V}_{\mathrm{Q}}(\mathrm{i})-\mathrm{V}_{\mathrm{M}}(\mathrm{i})\right|
$$


where $G$ is the number of global parameters (7 in this case), $\alpha$ and $\beta$ are the weights applied, respectively, to global parameters distance and to that of the histogram descriptor.

\subsection{Performance assessment}

Precision and Recall are two parameters commonly used to assess the performance of a retrieval system. They are given by relations (4) and (5)

$$
\begin{gathered}
\text { Precision }=\frac{\#(\text { Retrieved Relevant) }}{\text { \#Retrieved }} \\
\text { Recall }=\frac{\#(\text { Retrieved Relevant) }}{\text { \#Relevant }}
\end{gathered}
$$

The BEP indicates the percentage of the retrieved relevant contours after we have retrieved twice the number of relevant contours in the database: this corresponds to the Recall for the twice the number of relevant contours in the database. It provides an objective measurement of the efficiency of a retrieval system: the higher it is, the better is the system.

Results presented in the next sections correspond to the average values all over the database by using each contour of the database as a query.

\subsection{Test sets}

The evaluation is done on three databases. The first one is proposed by Klein and Kimia [8]. It consists of shapes gathered from different sources to form 9 different categories. Each category contains 11 shapes leading to variations in form, occlusion, articulation, missing parts... This gives a total of 99 shapes. Figure 5 illustrates this dataset. As our method utilizes contour-based analysis, an edge detector is applied to each of these elements to extract the corresponding contours.

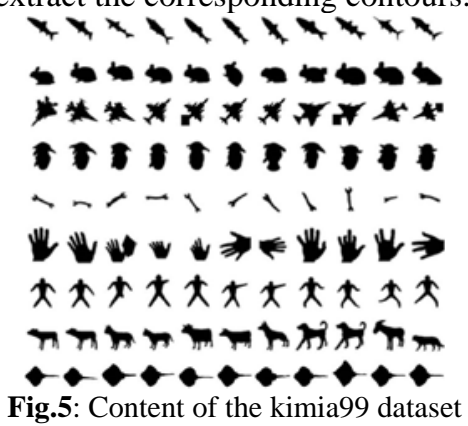

The second dataset is the multiview curve dataset (MCD) [14]. It consists of 40 classes drawn from the MPEG-7 Core Experiment Shape-1. Each class contains 14 contours corresponding to different perspective distortions of the original one. Figure 6 shows a sample set taken from this database. 


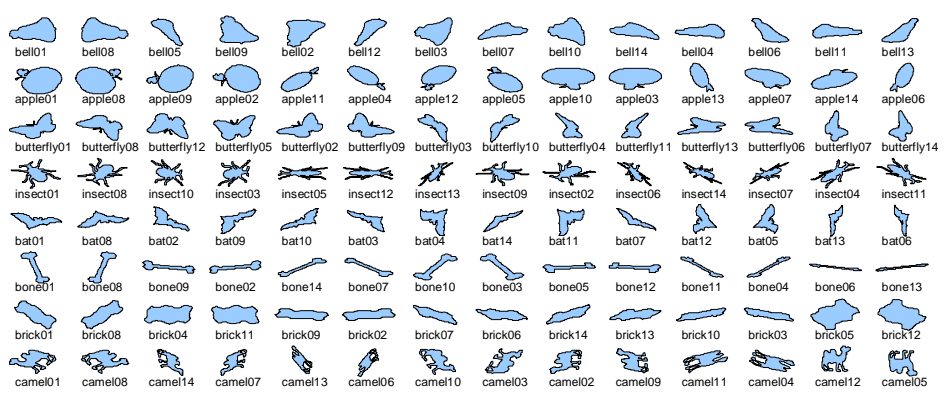

Fig.6: A sample set of MCD database contours

The MCD dataset presents realistic perspective transformations that one can encounter while creating images of real objects. Indeed, in the construction process, the authors [10] have printed the 40 original contours on white paper and for each one, they took images from 7 different view angles using a digital camera and then contours are extracted. By adding random rotations and reflections to these samples, the number of samples in each class is doubled to 14, leading to a total of 560 shapes in the database. The third dataset is the SQUID subset used by F. Mokhtarian and M. Bober in [9] and by F. Mokhtarian et al. in [12]. It consists of 17 classes containing from 6 to 8 shapes selected from the SQUID marine animals database [15] represented on Fig.7, containing 131 shapes.

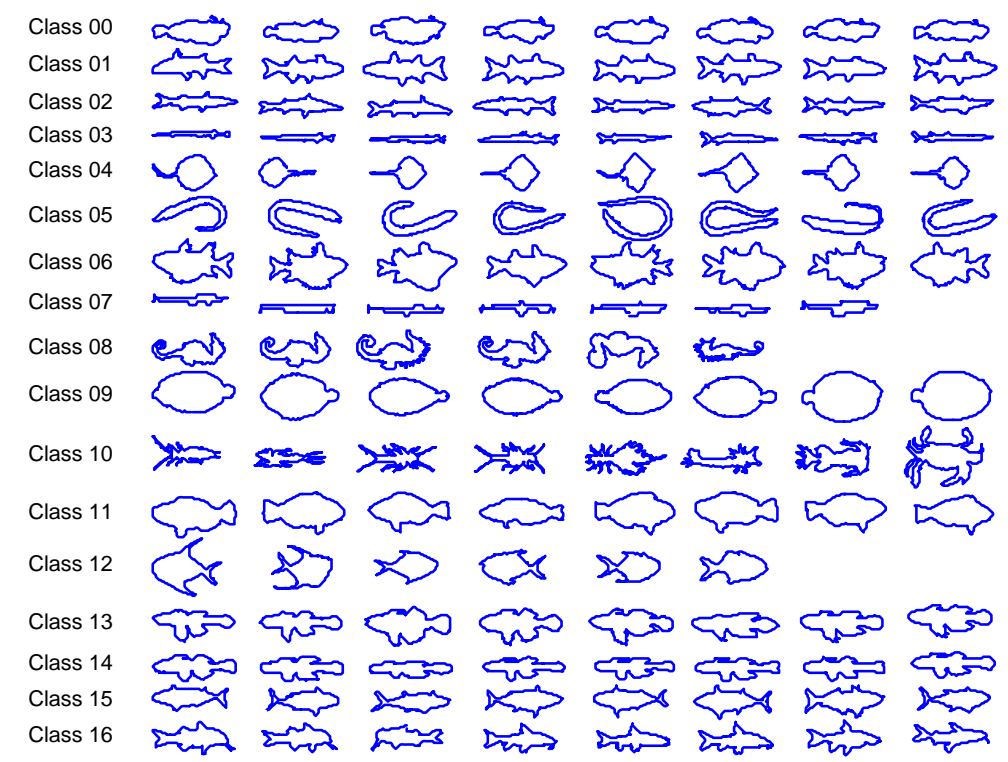

Fig.7: Dataset-III extracted from the SQUID database 
The evaluation on this database is done by using the same procedure as proposed in [9]. Similar to the concept of Recall, the procedure is as follows:

1. for a given class, use each of the contours as the query and determine the first 15 most similar outputs. Count the number of retrieved relevant contours and compute the ratio of this number to the number of relevant contours in the class. The mean value of this ratio all over the class is the performance measure for the concerned class (see individual columns in table III).

2. repeat 1 . for all the classes in the dataset. The overall performance is then obtained by averaging the performance provided by the 17 classes. Column 'Overall' in table III, gives that overall performance for the four involved methods.

\subsection{Experimental results}

In the experiments we have evaluated the effect of the length of the descriptor. This led us to observe that, for both histogram generation techniques, the performance varies a lot at the beginning and then establishes from a limit length above which there is no gain in performance.

\subsubsection{Comparison of the two histograms techniques}

Figure 8 demonstrates the BEP performance computed for a range of the number of bins. This figure shows a performance gap between both techniques of histogrambased descriptor generation. This gap is observed all over the three tested databases.
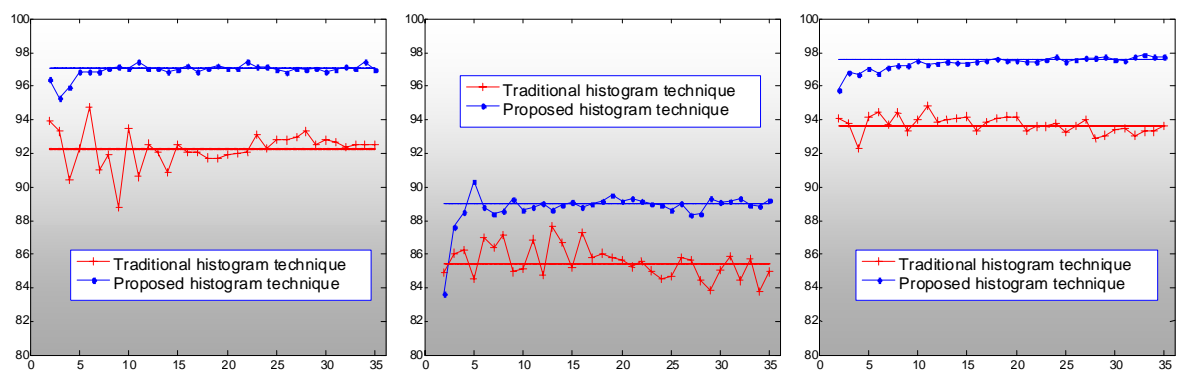

Fig.8: BEP vs. number of bins for a) kimia99 b) SQUID and c) MCD databases

Table I summarizes the retrieval results from the three databases. We present the mean, the standard deviation and the maximal values of BEP over the number of bins used. On the right of the table, we give the differences of those respective values between the two techniques: the value obtained from the new technique minus that of the traditional technique. From this table, it appears clearly that the proposed technique gives the best performance with more than 3.58\% mean BEP improvement. Notice also that the proposed technique gives more stable descriptor since the BEP values vary the least: we obtain negative difference values indicating standard deviation reduction (of more that 0.30 ). 
Table I: Comparison of the techniques.

\begin{tabular}{|c|c|c|c|c|c|c|c|c|c|}
\hline & \multicolumn{2}{|c|}{$\begin{array}{c}\text { Traditional histogram } \\
\text { generation (THi) }\end{array}$} & \multicolumn{3}{c|}{$\begin{array}{c}\text { Proposed histogram } \\
\text { generation (PHi) }\end{array}$} & \multicolumn{3}{c|}{ Difference (PHi-THi) } \\
\hline & kimia99 & SQUID & MCD & kimia99 & SQUID & MCD & kimia99 & SQUID & MCD \\
\hline Mean & 92.82 & 85.41 & 93.68 & 96.70 & 88.99 & 97.56 & 3.88 & 3.58 & 3.88 \\
\hline$\sigma$ & 0.66 & 0.97 & 0.45 & 0.18 & 0.29 & 0.15 & -0.48 & -0.68 & -0.30 \\
\hline Max & 94.95 & 87.63 & 94.82 & 97.34 & 90.35 & 97.87 & 2.39 & 2.72 & 3.05 \\
\hline
\end{tabular}

4.3.2 Comparison of the proposed approach with other approaches

In this subsection, we evaluate the proposed approach and compare it with some existing methods by applying it to appropriate databases.

A - Kimia99 dataset: in this experiment, we retrieve the top 15 most similar for each query. Figure 9 shows an example of this retrieval. The left column represents the query contour. As each category (or class) is composed of 11 contours, there are 10 relevant contours for each query: hence columns $1 \sim 10$ represent the first top 10 . This figure shows that, in most cases, most of the contours from the query category are among the first 10 retrieved contours. In general, the whole class is retrieved after 15 retrievals except for the $8^{\text {th }}$ category (row 8) that misses one shape. The BEP performance for this database is of $97.43 \%$ for 11 bins (leading to a 33-length descriptor). In a recent survey, Chandan Singh and Pooja [2] compared their methods with others and showed that their proposal outperforms the best on this dataset. Their proposed descriptor (ZM+HLTC) is hybrid and consists of two kinds of features: 1) local features extracted from the contour by using Hough transform and 2) global (region-based) features extracted from the region of the shape by using Zernike moments (ZM).

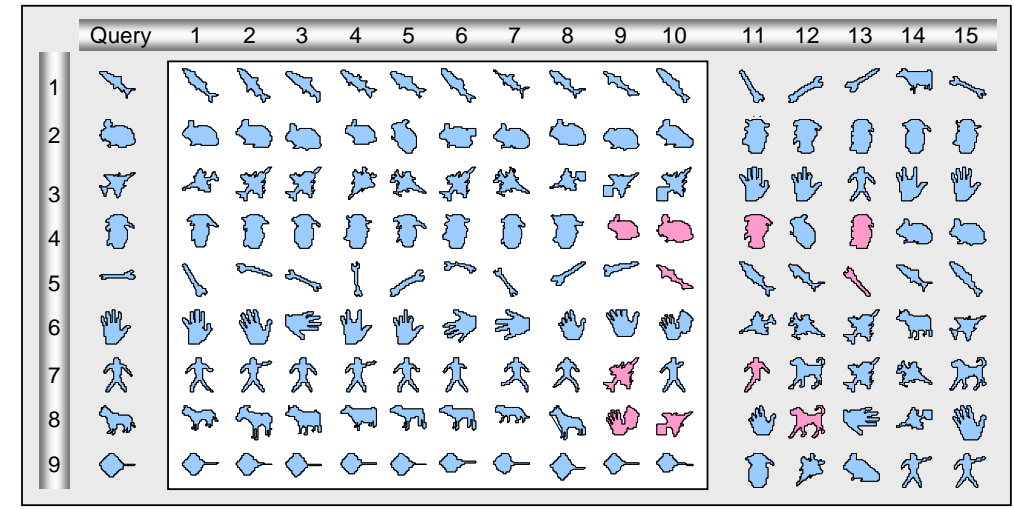

Fig.9: Results of retrieval from kimia99 database

The BEP obtained is $99.55 \%$ : this performance is very challenging. The comparison reported in [2] is done with methods using local, global or hybrid descriptors. Table II 
shows the performance of involved methods: as can be observed, the proposed method ranks second just after the challenging ZM+HLTC method. As our descriptor uses only local features, we can reasonably believe that we could improve our performance to meet the latter if we integrate global features.

Table II: Comparison of average BEP with other methods applied to kimia99.

\begin{tabular}{|c|c|c|c|c|c|c|c|c|c|}
\hline $\begin{array}{c}\text { FD } \\
\text { local }\end{array}$ & $\begin{array}{c}\text { WLD } \\
\text { local }\end{array}$ & $\begin{array}{c}\text { CPDH } \\
\text { local }\end{array}$ & $\begin{array}{c}\text { Ml } \\
\text { global }\end{array}$ & $\begin{array}{c}\text { GFD } \\
\text { global }\end{array}$ & $\begin{array}{c}\text { ZMD } \\
\text { global }\end{array}$ & $\begin{array}{c}\text { TCS } \\
\text { hybrid }\end{array}$ & $\begin{array}{c}\text { QLS } \\
\text { hybrid }\end{array}$ & $\begin{array}{c}\text { ZM+HLTC } \\
\text { hybrid }\end{array}$ & $\begin{array}{c}\text { Proposed } \\
\text { local }\end{array}$ \\
\hline 84.29 & 74.39 & 90.05 & 8.74 & 87.93 & 92.68 & 84.29 & 82.81 & 99.55 & $\mathbf{9 7 . 4 3}$ \\
\hline
\end{tabular}

B - SQUID dataset: the following table shows the retrieval results from the SQUID database. For comparison purpose, we use results from the well-known curvature scale space (CSS) descriptor, the Fourier descriptor (FD) and the moment invariants (MI) reported in [9]. Columns 1 17 indicate the mean success retrieval rate for each class and the column "Overall" indicates the mean success retrieval rate over the whole database. As can be seen on this table, the proposed approach outperforms the FD and MI descriptors and ranks first (ex aequo with CSS method).

Table III: Comparison of retrieval rate with other methods applied to SQUID dataset

\begin{tabular}{|c|c|c|c|c|c|c|c|c|c|c|c|c|c|c|c|c|c|c|c|}
\hline \multicolumn{2}{|c|}{ Class $\mathrm{N}^{\circ}$} & 1 & 2 & 3 & 4 & 5 & 6 & 7 & 8 & 9 & 10 & 11 & 12 & 13 & 14 & 15 & 16 & 17 & Overall \\
\hline \multirow{4}{*}{ 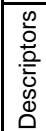 } & CSS & 89 & 86 & 81 & 95 & 100 & 100 & 91 & 98 & 75 & 92 & 81 & 100 & 94 & 89 & 100 & 95 & 69 & 91 \\
\hline & Proposed & 88 & 91 & 96 & 100 & 100 & 97 & 93 & 98 & 75 & 97 & 74 & 96 & 92 & 85 & 91 & 99 & 68 & 91 \\
\hline & FD & 100 & 84 & 100 & 75 & 78 & 42 & 80 & 100 & 36 & 98 & 13 & 70 & 78 & 98 & 73 & 97 & 48 & 75 \\
\hline & $\mathrm{MI}$ & 34 & 78 & 91 & 100 & 88 & 41 & 72 & 86 & 47 & 53 & 23 & 91 & 53 & 45 & 72 & 73 & 58 & 65 \\
\hline
\end{tabular}

C - MCD dataset: Figure 10 shows the Precision vs. Recall graphs obtained from the MCD database. Compared to the results reported in recent studies [3] and [11], the proposed approach performs very well. The Recall-Precision graph indicates more than 91.86\% Recall for more than 92\% Precision for the proposed approach. This outperforms the reported performance that are $86 \%$ Recall for $85 \%$ Precision for Helmholtz curve descriptor (HCD) [11] and 90\% Recall for 90\% Precision obtained by Ekombo et al. with the invariant Fourier descriptor [3].

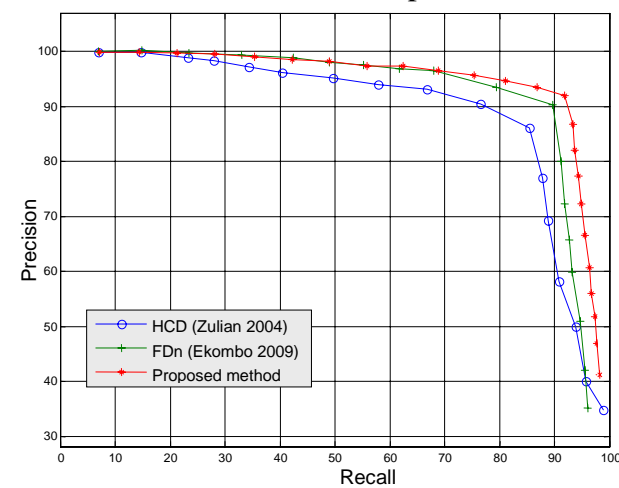

Fig.10: Example of Precision vs. Recall graphs. 


\section{Conclusion}

The subject of this study is the description of planar objects based on the analysis of their contours. For this purpose, we introduced two contributions: 1) first, we proposed a new descriptor based on the histograms of three features derived from the turning angle scale space analysis of the contours and 2) we adopted another technique for histogram descriptor generation as opposed to traditional histogram-based descriptors. The proposed technique of histogram generation jointly with the new features provides an efficient and effective descriptor that outperforms the traditional histogram descriptor. Applied to three databases, the proposed technique gave more than $3.58 \%$ improvement of the bull's eye performance regarding the traditional histogram descriptors.

Initially designed for d-TASS-based descriptor for shape retrieval, the novel technique of histogram-based descriptor construction is successfully applied for texture retrieval. These encouraging results suggest a potential application (using an appropriate set of features: DCT, ...) of the novel proposed histogram technique on medical images retrieval.

Finally, as demonstrated by experimental results, the proposed descriptor allows reaching state-of-the-art performance. Since this descriptor is based only on contour information, we believe that the descriptor can be improved by combining it with a region-based feature.

From the previous observations, the future developments of this study will be of two main types: the first will be to generate a hybrid descriptor by combining contourbased and region-based features in order to improve the shape descriptor and the second will be to extend the proposed histogram generation technique to medical images description for their retrieval from databases.

Acknowledgements: This work was partially supported by the Franco-Algerian cooperation program PHC TASSILI grants. Entitled "Système Conjoint de Compression et d'Indexation Basé-Objet pour la Vidéo (SCCIBOV)", the project gathers members of Institut National des Sciences Appliquées (INSA) de Rennes, Université de Technologie de Compiègne (UTC), the University of Sidi Bel Abbès and the University of Mascara.

\section{References}

[1] Bai, C., Kpalma K., and Ronsin, J., A new descriptor based on 2D DCT for image retrieval, International Conference on Computer Vision Theory and Applications (VISAPP), pp. 714-717, February 24-26, 2012, Roma, Italy

[2] Chandan Singh, Pooja, Improving image retrieval using combined features of Hough transform and Zernike moments, Elsevier, Optics and Lasers in Engineering, Vol. 49, 
No.12, pp 1384-1396, 2011

[3] Ekombo, P. L. E., Ennahnahi, N., Oumsis, M., and Meknassi, M., Application of affine invariant Fourier descriptor to shape-based image retrieval, International Journal of Computer Science and Network Security (IJCSNS), Vol.9, No.7, pp. 240-247, 2009

[4] Kpalma, K., Yang, M. and Ronsin, J., Planar shapes descriptors based on the turning angle scalogram, 5th International Conference on Image Analysis and Recognition (ICIAR), Povoa de Varzim, Portugal, June 25-27, pp. 547-556, Proceedings, Lecture Notes in Computer Science, Vol.5112, Springer, ISBN 978-3-540-69811-1, 2008

[5] Kpalma, K., and Ronsin, J., Turning Angle Based Representation for Planar Objects, Electronics Letters / IEE Electronics Letters, Vol.43, No.10, pp. 561-563, 2007

[6] Kpalma, K., and Ronsin, J., Multiscale contour description for pattern recognition, Pattern Recognition Letters, Vol.27, No.13, pp. 1545-1559, 1 October 2006

[7] Zhong D., Defée I. DCT histogram optimization for image database retrieval, Pattern Recognition Letters, Vol. 26, pp. 2272-2281, 2005.

[8] Klein, S.T.B., P.N., Kimia, B.B. Recognition of shapes by editing their shock graphs. IEEE Trans. Pattern Anal. Machine Intell. Vol.26, No.5, pp. 550-571, 2004.

[9] Zhang, D., and Lu, G. Review of shape representation and description techniques, Pattern Recognition, Vol. 37, pp. 1-19, 2004.

[10] Zuliani, M., Bhagavathy, S., Manjunath, B., and Kenney, C. S., Affine-invariant curve matching, in IEEE International Conference on Image Processing (ICIP), October 2004.

[11] Zuliani, M., Kenney, C., Bhagavathy, S., Manjunath, B. S., Drums and Curve Descriptors, British Machine Vision Conference (BMVC), September 2004.

[12] Mokhtarian, F., and Bober, M., Curvature Scale Space Representation: Theory, Applications and MPEG-7 Standardization. Kluwer Academic Publishers, 2003.

[13] Mokhtarian F. and A. K Mackworth. A Theory of Multiscale, Curvature-Based Shape Representation for Planar Curves, in IEEE Transactions on Pattern Analysis and Machine Intelligence, Vol. 14, №. 8, pp. 789 - 805, 1992

[14] http://vision.ece.ucsb.edu/ Zuliani/Research/MCD/MCD.shtml, visited November 25, 2010

[15] http://www.ee.surrey.ac.uk/CVSSP/demos/css/demo.html, visited November 25, 2010 